Review

\title{
Supplementation with the Leucine Metabolite $\beta$-hydroxy- $\beta$-methylbutyrate (HMB) does not Improve Resistance Exercise-Induced Changes in Body Composition or Strength in Young Subjects: A Systematic Review and Meta-Analysis
}

\author{
Josephine S. Jakubowski ${ }^{1}{ }^{(}$, Everson A. Nunes ${ }^{1,2}{ }^{\oplus}$, Filipe J. Teixeira ${ }^{3}{ }^{(}$, Victoria Vescio ${ }^{1}$, \\ Robert W. Morton ${ }^{1}$, Laura Banfield ${ }^{4}(\mathbb{D})$ and Stuart M. Phillips ${ }^{1, *(D)}$ \\ 1 Department of Kinesiology, McMaster University, Hamilton, ON L8S 4L8, Canada; \\ vesciojs@mcmaster.ca (J.S.J.); nunese1@mcmaster.ca (E.A.N.); vvescio11@gmail.com (V.V.); \\ mortonrw@mcmaster.ca (R.W.M.) \\ 2 Department of Physiological Sciences, Federal University of Santa Catarina, Florianópolis, \\ SC 88040-900, Brazil \\ 3 CBIOS-Universidade Lusófona's Research Center for Biosciences and Health Technologies, Campo Grande, \\ 1749024 Lisboa, Portugal; ftperformancenutrition@gmail.com \\ 4 Health Sciences Library, McMaster University, Hamilton, ON L8S 4L8, Canada; banfie@mcmaster.ca \\ * Correspondence: phillis@mcmaster.ca; Tel.: +1-905-525-9140 (ext. 24465)
}

Received: 8 May 2020; Accepted: 21 May 2020; Published: 23 May 2020

\begin{abstract}
HMB) is a leucine metabolite that is purported to increase fat-free mass (FFM) gain and performance in response to resistance exercise training (RET). The aim of this systematic review and meta-analysis was to determine the efficacy of HMB supplementation in augmenting FFM and strength gains during RET in young adults. Outcomes investigated were: total body mass (TBM), FFM, fat mass (FM), total single repetition maximum (1RM), bench press (BP) 1RM, and lower body (LwB) 1RM. Databases consulted were: Medical Literature Analysis and Retrieval System Online (Medline), Excerpta Medica database (Embase), The Cumulative Index to Nursing and Allied Health Literature (CINAHL), and SportDiscus. Fourteen studies fit the inclusion criteria; however, 11 were analyzed after data extraction and funnel plot analysis exclusion. A total of 302 participants (18-45 y) were included in body mass and composition analysis, and 248 were included in the strength analysis. A significant effect was found on TBM. However, there were no significant effects for FFM, FM, or strength outcomes. We conclude that HMB produces a small effect on TBM gain, but this effect does not translate into significantly greater increases in FFM, strength or decreases in FM during periods of RET. Our findings do not support the use of HMB aiming at improvement of body composition or strength with RET.
\end{abstract}

Keywords: muscle mass; resistance exercise; strength training; HMB; 1RM; hypertrophy; fat-loss

\section{Introduction}

$\beta$-hydroxy- $\beta$-methylbutyrate (HMB) is a metabolite derived from the essential amino acid leucine [1]. Some research suggests that $\mathrm{HMB}$ is an anabolic compound that increases resistance exercise training-(RET)-induced gains in fat-free mass (FFM) [2-9]. Also, many studies have been conducted to examine the impact of HMB on body fat loss and muscle strength and performance-related outcomes $[7,10-12]$. Some studies using rodents $[13,14]$ and non-exercising humans $[15,16]$ have shown that HMB was able to modulate some aspects that might be directly or indirectly linked to skeletal 
muscle hypertrophy. For example, HMB has been shown to stimulate muscle protein synthesis in cells in vitro [17]. Also, in vitro [18] and rodent studies [19] have shown that HMB can decrease protein catabolism by modulating the activity of proteins involved in both protein synthesis and protein breakdown. As a nutritional supplement, HMB is available in two forms: a calcium-bound (HMB-Ca) form and a free-acid (HMB-FA) form [15,16]. It has been shown that $3 \mathrm{~g}$ of HMB-FA [15] and $3 \mathrm{~g}$ of HMB-Ca [16] acutely stimulate MPS to a similar extent in healthy young men.

Several reviews have been published citing the efficacy of $\mathrm{HMB}$ as a strategy to improve changes in body composition and/or performance during resistance exercise [20-23]. However, systematic reviews and meta-analyses are equivocal in support of the anabolic properties of HMB (Table 1). For example, Rowlands and Thomson [7] conducted a systematic review and meta-analysis in which they examined the HMB effects in trained and untrained subjects undergoing RET. The only significant finding of their meta-analysis was a small improvement in leg strength in untrained subjects, but no significant effects on body composition [7]. Similarly, a meta-analysis performed to test the effects of $\mathrm{HMB}$ on body composition and strength of trained and competitive athletes found no beneficial effects of HMB supplementation [11]. However, Silva et al., [24], examining only HMB-free acid (HMB-FA), concluded that HMB-FA ingestion for 12 weeks enhanced resistance training-induced increases in total body mass and fat-free mass and fat mass loss. Also, the authors concluded that HMB-FA ingestion promoted higher gains of strength and better performance in high-intensity assessments such as the Wingate, and vertical jump test [24]. Recently, Fernandez-Landa et al., [25] conducted a systematic review including only studies using the combination of HMB and creatine and concluded that HMB might have some potential effects on body composition. Nevertheless, the effect of creatine on body composition, well known to have a positive effect on body composition and strength gains [26], could not be ruled out as the sole reason for the effects observed separate from a potential effect of HMB. In sum, various meta-analyses provide disparate answers to the question of the effectiveness of $\mathrm{HMB}$ in enhancing body composition (increased lean mass and reduced fat mass) as well as strength during RET. 
Table 1. Main findings of previous meta-analyses assessing effects of $\beta$-hydroxy- $\beta$-methylbutyrate (HMB) ingestion in lean body mass and strength gains in subjects submitted to a resistance exercise program.

\begin{tabular}{|c|c|c|c|c|c|c|c|c|c|c|}
\hline \multirow{2}{*}{ Study } & \multirow{2}{*}{ Objective } & \multirow{2}{*}{ Criteria } & \multirow{2}{*}{$\begin{array}{l}\text { Included } \\
\text { Studies }\end{array}$} & \multirow{2}{*}{ Supplement } & \multirow{2}{*}{ Outcome } & \multirow{2}{*}{ Conclusion } & \multirow{2}{*}{ Lean Mass } & \multirow{2}{*}{ Strength } & \multicolumn{2}{|c|}{ Effect Size } \\
\hline & & & & & & & & & Lean Mass & Strength \\
\hline $\begin{array}{l}\text { Nissen and Sharp } \\
\text { (2003) [27] }\end{array}$ & $\begin{array}{l}\text { To quantify } \\
\text { dietary supplements } \\
\text { to augment lean mass } \\
\text { and strength gains } \\
\text { during Resistance } \\
\text { Exercise } \\
\text { Training (RET) }\end{array}$ & $\begin{array}{l}\text { Randomized } \\
\text { clinical trial } \\
\text { (RCT) } \\
\text { Duration: } \\
\text { Full Body RET } \\
\geq 3 \mathrm{wk} \\
\text { Frequency: } \\
\geq 2 \mathrm{x} / \mathrm{wk}\end{array}$ & $\begin{array}{l}9 \text { studies } \\
\text { - Nissen et al. } \\
\text { (1996) [2] } \\
\text { included as } \\
2 \text { studies }\end{array}$ & $3 \mathrm{~g} \mathrm{HMB}-\mathrm{Ca}$ & $\begin{array}{l}\text { Lean Mass } \\
\text { Total Strength }\end{array}$ & $\begin{array}{l}\text { There is sufficient data to } \\
\text { support the use of HMB } \\
\text { to augment lean mass and } \\
\text { strength gains with RET }\end{array}$ & $\begin{array}{l}\text { HMB results in an } \\
\text { increase of } 0.28 \% / \text { wk } \\
\text { confidence interval } \\
\text { (CI): } 0.13 \% \text { to } 0.42 \%\end{array}$ & $\begin{array}{l}\text { HMB results in an } \\
\text { increase of } 1.40 \% / \mathrm{wk} \\
\text { CI: } 0.41 \% \text { to } 2.39 \%\end{array}$ & $\begin{array}{l}\text { Trivial } \\
\text { effect size (ES): } \\
0.15 \text { CI: } 0.06 \text { to } \\
0.24 p<0.005\end{array}$ & $\begin{array}{l}\text { Trivial } \\
\text { ES: } 0.19 \text { CI: } \\
0.09-0.29 \\
p<0.01\end{array}$ \\
\hline $\begin{array}{l}\text { Rowlands and } \\
\text { Thomson } \\
\text { (2007) [7] }\end{array}$ & $\begin{array}{l}\text { To meta-analyze the } \\
\text { effectiveness of HMB } \\
\text { on strength, body } \\
\text { composition, and } \\
\text { muscle damage in } \\
\text { trained (TR) and } \\
\text { untrained (UT) } \\
\text { participants } \\
\text { during RET }\end{array}$ & $\begin{array}{l}\text { RCT } 8 / 9 \\
\text { Crossover 1/9 } \\
\text { TR or UT men } \\
\text { No criteria for } \\
\text { duration }\end{array}$ & $\begin{array}{l}9 \text { studies } \\
\mathrm{N}=394\end{array}$ & $\begin{array}{l}1 / 9 \\
\text { HMB + drink } \\
\text { (whey } \\
\text { protein-carbohydrate, } \\
\text { vitamins, minerals, } \\
\text { glutamine, and } \\
\text { chromium picolinate) } \\
\text { 1/9 HMB+ glucose+ } \\
\text { taurine+ disodium } \\
\text { phosphate+ potassium } \\
\text { phosphate } \\
\text { 2/9 HMB + } 50 \mathrm{mg} \text { of } \\
\text { potassium phosphate }\end{array}$ & $\begin{array}{l}\text { Lean Mass } \\
\text { Strength } \\
\text { Creatine } \\
\text { kinase (CK) }\end{array}$ & $\begin{array}{l}\text { HMB } \\
\text { supplementation results in a } \\
\text { small beneficial increase to } \\
\text { overall strength in UT lifters } \\
\text { but has a negligible effect } \\
\text { on TR lifters. } \\
\text { In UT participants HMB } \\
\text { results in a small increase in } \\
\text { lower-body } \\
\text { strength, } \\
\text { In both trained and untrained } \\
\text { lifters, the effect } \\
\text { of HMB supplementation on } \\
\text { body composition is negligible. }\end{array}$ & $\begin{array}{l}\text { Fat-free mass (FFM) } \\
\text { increases and changes } \\
\text { in fat mass (FM) in UT } \\
\text { and TR lifters were } \\
\text { negligible }\end{array}$ & $\begin{array}{l}\text { UT: } \\
\text { Small benefit } \\
\text { Lower-body: } \\
9.9 \% \pm 5.9 \% \\
\text { Average strength: } \\
6.6 \% \pm 5.7 \% \\
\text { Negligible gains } \\
\text { Upper-body strength: } \\
2.1 \% \pm 5.5 \% \\
\text { TR: All outcomes } \\
\text { Trivial } \\
\text { UT and TR combined: } \\
\text { Trivial } \\
\text { Average strength: } \\
3.7 \% \pm 2.4 \%\end{array}$ & $\begin{array}{l}\text { Trivial } \\
\text { 3-unit increase } \\
\text { of HMB daily } \\
\text { dose } \\
\text { ES: } \\
-0.07 \pm 0.17\end{array}$ & $\begin{array}{l}\text { Trivial } \\
\text { 3-unit increase } \\
\text { of HMB daily } \\
\text { dose } \\
\text { ES: } 0.02 \pm 0.2\end{array}$ \\
\hline $\begin{array}{l}\text { Sanchez-Martinez } \\
\text { et al. (2018) [11] }\end{array}$ & $\begin{array}{l}\text { Examine the } \\
\text { effectiveness of HMB } \\
\text { supplementation on } \\
\text { strength and body } \\
\text { composition in } \\
\text { trained athletes }\end{array}$ & $\begin{array}{l}\text { RCT or } \\
\text { cross-over } \\
\text { TR }(\geq 1 \text { RET) } \\
\text { or competitive } \\
\text { athletes }\end{array}$ & $\begin{array}{l}6 \text { studies } \\
\text { - Slater et al. } \\
\text { (2001) [6] } \\
\text { included as } 4 \\
\text { studies } \\
\text { - Kreider et al. } \\
\text { (1999) [3] } \\
\text { included as } \\
\text { 2 studies }\end{array}$ & $\begin{array}{l}\text { 5/6 RCT } \\
1 / 6 \text { crossover } \\
\text { 4/6 } \mathrm{g} \mathrm{HMB-Ca} \\
\text { 2/6 did not specify } \\
\text { HMB-FA/HMB-Ca } \\
\text { 1/6 HMB + drink } \\
\text { (proteins, } \\
\text { carbohydrates, } \\
\text { vitamins, minerals) } \\
1 / 7 \text { HMB+ L-Carnitine, } \\
\text { Choline, Boron and } \\
\text { Garcinia Cambogia } \\
\end{array}$ & $\begin{array}{l}\text { Body mass } \\
\text { Fat-free mass } \\
\text { Fat mass } \\
\text { Bench } \\
\text { Leg press }\end{array}$ & $\begin{array}{l}\text { No effect of HMB on strength } \\
\text { and body composition in } \\
\text { competitive athletes }\end{array}$ & $\begin{array}{l}\text { HMB has a negligible ef } \\
\text { composition and streng } \\
\text { competitive athletes }\end{array}$ & $\begin{array}{l}\text { fect on body } \\
\text { th in trained and }\end{array}$ & $\begin{array}{l}\text { Body mass ES } \\
=-0.01, \mathrm{CI}: \\
-0.29 \text { to } 0.27 \\
\text { Fat free mass } \\
\mathrm{ES}=0.16, \mathrm{CI}: \\
-0.5 \text { to } 0.46\end{array}$ & $\begin{array}{l}\text { Bench press } \\
\text { ES }=0.0, \text { CI: } \\
-0.32 \text { to } 0.32 \\
\text { Leg press ES }= \\
-0.09, \text { CI: } 0.46 \\
\text { to } 0.28\end{array}$ \\
\hline $\begin{array}{l}\text { Holland et al. } \\
2019 \text { [28] }\end{array}$ & $\begin{array}{l}\text { Determine the effects } \\
\text { of HMB on body } \\
\text { composition in } \\
\text { athletes }\end{array}$ & $\begin{array}{l}\mathrm{RCT} \\
\text { Minimum } \\
\text { supplement } \\
\text { period of } 4 \mathrm{wk}\end{array}$ & $\begin{array}{l}\text { Body Mass: } 7 \\
\text { studies, } N=208 \\
\text { Fat Free and Fat } \\
\text { Mass } 5 \text { studies, } \\
N=161 \text { and } N= \\
128, \text { respectively }\end{array}$ & $\begin{array}{l}\text { HMB } \\
\text { * Does not differentiate } \\
\text { HMB-Ca vs. HMB-FA }\end{array}$ & $\begin{array}{l}\text { Body Mass } \\
\text { Fat Free Mass } \\
\text { Fat Mass }\end{array}$ & $\begin{array}{l}\text { HMB may have a small, } \\
\text { positive impact on FFM in } \\
\text { athletes when protein intake is } \\
\text { suboptimal ( }<1.6 \mathrm{~g} / \mathrm{kg} / \mathrm{day})\end{array}$ & $\begin{array}{l}\text { There was no significan } \\
\text { although the CI was ske } \\
\text { small effect } \\
\text { There was no significan }\end{array}$ & $\begin{array}{l}\text { effect of HMB on FFM, } \\
\text { wed in favor of a } \\
t \text { effect of HMB on BM }\end{array}$ & $\begin{array}{l}\mathrm{ES}=-0.30 \pm \\
0.13 ; 95 \% \mathrm{CI}: \\
20.07 \text { to } 0.68 \\
(p=0.00)\end{array}$ & $\begin{array}{l}\text { Body Mass } \\
\text { ES }=20.02 \pm \\
0.04 ; 95 \% \text { CI: } \\
20.14 \text { to } 0.10 \\
(p=0.70)\end{array}$ \\
\hline
\end{tabular}

Abbreviations: $\mathrm{CI}=$ Confidence Interval; $\mathrm{CK}=$ Creatine Kinase; ES = Effect Size; FFM = Fat free mass; FM = Fat mass; RCT = randomized clinical trial; RET = Resistance Exercise Training; $\mathrm{TR}=$ Trained; $\mathrm{UT}=$ Untrained 
Here, we sought to conduct a comprehensive systematic review and meta-analysis on HMB with RET in untrained and trained persons. Based on a lack of any discernable difference between the HMB-FA [15] and HMB-Ca [16] forms of the supplement in terms of anabolic properties, despite an ostensibly higher bioavailability of the FA-form versus the Ca-form of HMB [29], we aimed to study both forms of HMB in this analysis. Our aim was a systematic review and meta-analysis to bring an updated evidence-based answer to whether or not HMB supplementation augments resistance exercise-induced gains in fat-free (i.e., lean) mass, reductions in body fat mass (FM), and increases in strength.

\section{Materials and Methods}

This systematic review and meta-analysis were performed according to the Cochrane Handbook for Systematic Reviews of Interventions [30] and is reported according to the PRISMA (Preferred Reporting Items for Systematic Reviews and Meta-Analysis) guidelines [31].

\subsection{Eligibility Criteria}

Studies were eligible for inclusion if they met the following criteria according to the PICOS (Participants, Intervention, Control, Outcome measurements, and Study design) strategy. Participants were healthy untrained or trained men or women between the age range of 18-50 years old. The intervention dose of HMB was set at $3.0 \mathrm{~g} \mathrm{HMB} /$ day and study participants had to be conducting full body RET for $\geq 3$ weeks (training sessions at least twice/week). Comparators were any placebo and the same resistance exercise program. Outcomes were total body mass (TBM), fat-free (i.e., lean) body mass (FFM), fat mass (FM), and 1RM strength. Study designs included only double-blinded-randomized clinical trials.

\subsection{Systematic Search Strategy}

A literature search for randomized controlled trials (RCT) investigating the effect of HMB on lean body mass, strength in adult male subjects was conducted by electronic searching of relevant literature databases. Two investigators (JSJ and LB) performed database searchers (last search date 1 April 2020) on the Medical Literature Analysis and Retrieval System Online (Medline), Excerpta Medica database (Embase), The Cumulative Index to Nursing and Allied Health Literature (CINAHL) and SportDiscus using a predetermined search strategy based on relevant keywords (see Supplementary material for the search strategy). Limits were applied to the electronic search, restricting studies to those including adults and humans, and published in the English language only. Abstracts and conference proceedings were not included in the present meta-analysis.

\subsection{Data Extraction and Outcome Measures}

Studies were carefully reviewed and searched for information regarding the study design, the RET intervention, subject characteristics, supplement information, placebo/control information, body composition outcomes and performance outcomes, and any other notable information (e.g., sources of bias/conflict of interest). Data was independently extracted from the selected papers by three investigators (JSJ, FJT, and VV). For some studies, corresponding authors were contacted and asked to provide raw data. The following outcomes were investigated: TBM (measured by any scale); FFM, and muscle mass (i.e., lean mass) if FFM was not available (measured by dual-energy X-ray absorptiometry (DXA), hydrodensitometry, or whole-body air plethysmography (BodPod ${ }^{\circledR}$ ); and fat mass (FM; measured by DXA, hydrodensitometry and/or BodPod ${ }^{\circledR}$ ). Performance outcomes were: one-repetition-maximum (isotonic) strength (1RM; measured by any 1RM strength test). For strength, bench press $(\mathrm{BP}) 1 \mathrm{RM}$, and lower body $(\mathrm{LwB}) 1 \mathrm{RM}$ were extracted. LwB strength included leg press or squat exercises depending on which was used in the study. 


\subsection{Risk of Bias Analysis and Sensitivity Analysis}

Risk of bias was assessed independently by two investigators (JSJ and FJT) according to the Cochrane collaboration risk-of-bias tool (Cochrane Handbook for Systematic Reviews of Interventions version 6.0) [30]. Studies were carefully reviewed for details, including randomization methods, participant allocation, and blinding of the subjects and researchers directly involved with the subjects or data analysis. Also, incomplete outcome data, selective reporting, and other sources of bias were assessed. Where disagreements between the two investigators (JSJ and FJT) were not resolved by consensus on study eligibility, data extraction, and risk-of-bias assessment, a third investigator reviewed the paper to yield a decision (EAN). Studies not reporting randomization or blinding procedures were considered high risk in the domain allocation concealment and blinding of participants and personnel. Industry-related sponsorship or authorship were considered "unclear risk of bias" and included in "other bias". Funnel plots were generated to assess for evidence of asymmetry and potential publication bias [32]. Heterogeneity between studies was determined by $\mathrm{I}^{2}$, with values of $<50 \%$ considered low, $50-74.9 \%$ considered moderate, and 75-100\% considered high heterogeneity. Sensitivity analyses were also conducted. Studies assessed with 3 or more domains judged as high or unclear risk of bias were submitted to sensitivity analyses. These analyses were conducted for all outcomes by the "remove 1 " technique. Such a procedure aimed to assess whether individual studies had a disproportionate effect on the results of the meta-analyses [33].

\subsection{Data Syntheses and Meta-Analysis}

Only pre-intervention and post-intervention outcome data were retrieved if a study had multiple time points. Where the SD for change $(\Delta S D)$ was available, it was collected alongside the preand post-intervention SD. Studies testing HMB-Ca and HMB-FA forms had results of both groups combined using the RevMan calculator [34] (RevMan, V.5.3. Copenhagen: The Nordic Cochrane Centre, The Cochrane Collaboration, 2014) and then compared to placebo groups. When necessary, missing $\triangle S D$ was imputed according to the instructions of the Cochrane handbook using correlation coefficients obtained from studies were standard deviation for changes were presented. This procedure was necessary for four studies during body mass and composition analysis and for nine studies for strength data. The change in mean ( $\triangle$ Mean) and $\Delta S D$ were calculated for each group and uploaded to Review Manager [34] (RevMan, V.5.3. Copenhagen: The Nordic Cochrane Centre, The Cochrane Collaboration, 2014). Total strength 1RM was independently calculated by combining bench press and leg press, leg extension or squat 1RM using the RevMan calculator [34] (Rev Man V.5.3. Copenhagen: The Nordic Cochrane Centre, The Cochrane Collaboration, 2014). Random-effects meta-analyses were performed in RevMan on the change in each outcome of interest. Effect sizes are presented as mean difference $(\mathrm{MD})$ with means $\pm \mathrm{SD}$ and $95 \%$ confidence intervals $(\mathrm{CI})$. Test for overall effect (z score) was regarded significant when $p \leq 0.05$.

\section{Results}

\subsection{Included Studies}

A total of 1731 results were obtained from the search strategy and additional searches during data analysis (Figure 1). After removing duplicates, in vitro studies, articles not in English, and reviews, 1294 records were available for title and abstract screening. Of these, 303 records were retrieved for full-text screening with 14 double blinded-RCTs being eligible for inclusion (Table S1).

\subsection{Risk of Bias and Asymmetry Analysis}

After selecting studies according to the inclusion and exclusion criteria, we conducted a sensitivity analysis followed by risk of bias analysis. One study was removed due to missing data [3] (Table S2). Two additional studies were excluded (Table S2) after visual funnel plots symmetry analysis (Figure S1A) [35,36]. No asymmetry was detected when considering the remaining 
studies [2,4-6,8-10,12,37-39] (Figure S1B). One study received high risk of bias classification in three domains (selection bias, performance bias and other bias) [2]. Seven studies received an unclear risk classification for detection bias (blinding of the outcome assessment), due to lack of detailed description in the respective papers (Figure S2A,B) [2,4-6,10].
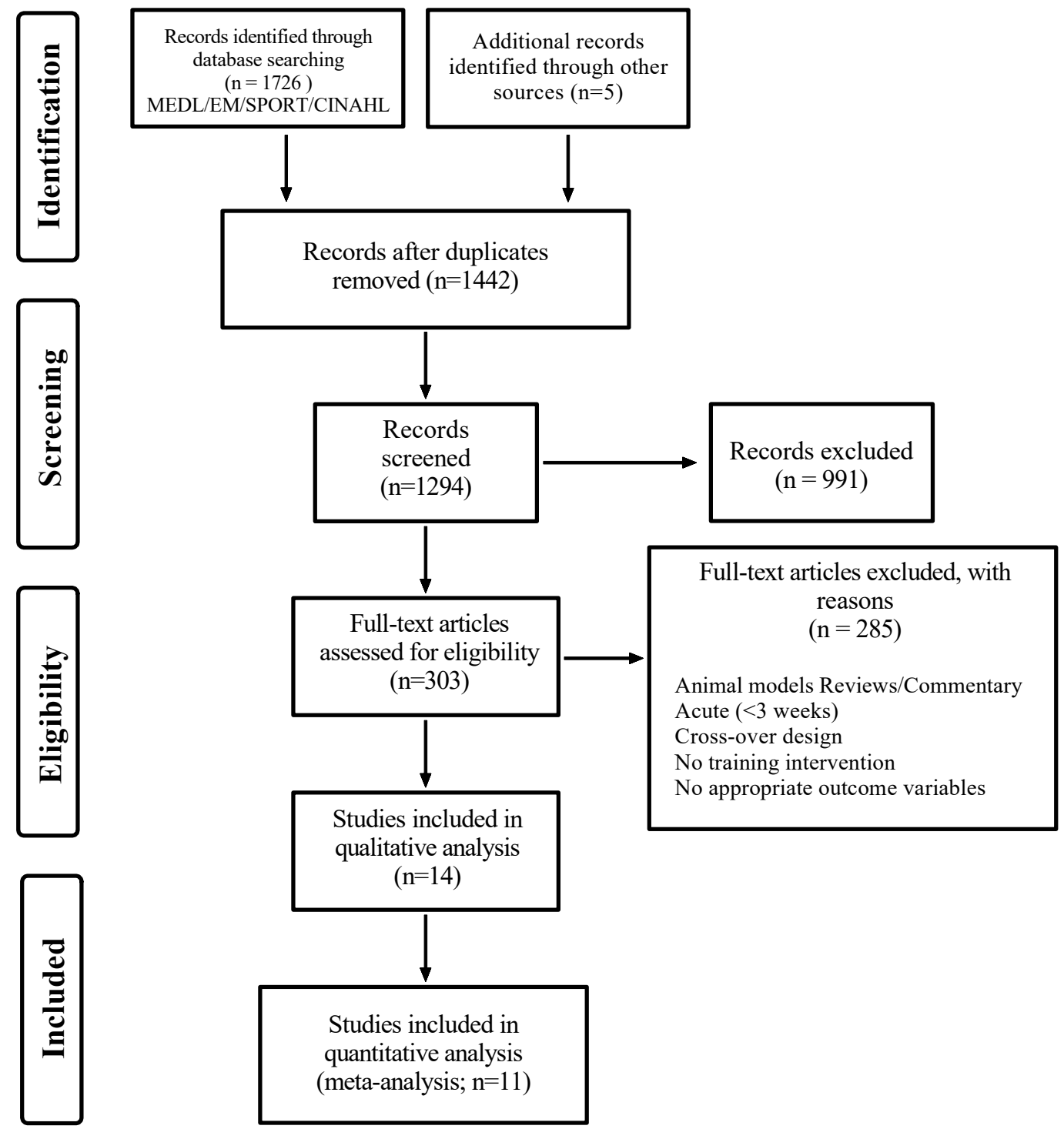

Figure 1. Preferred Reporting Items for Systematic Reviews and Meta-Analysis (PRISMA) flow chart. Medline: Medical Literature Analysis and Retrieval System Online; Embase: Excerpta Medica database; CINAHL: The Cumulative Index to Nursing and Allied Health Literature.

\subsection{Study Characteristics}

After removing studies according to sensitivity analysis, 11 studies remained to be meta-analyzed (Table 2). For the analysis, one article was considered as two separate RCT because data from men and women were available and permitted the inclusion as independent data [4]. A total of 302 participants were included in body mass and composition analysis, and 248 were included in the muscle strength analysis. The average age of the participants in the studies was 27 years (18-45 years). Study duration was $7.6 \pm 4.0$ weeks with a training frequency varying between $2-5$ days/week. Placebo was mainly carbohydrate based (e.g., rice flour, corn starch, polydextrose or microcrystalline cellulose) for most of the selected studies (Table 2). 
Table 2. Characteristics of the analysed studies.

\begin{tabular}{|c|c|c|c|c|c|c|c|c|c|c|c|c|c|c|c|}
\hline \multirow{3}{*}{ Study } & \multirow{3}{*}{ Country } & \multirow[t]{3}{*}{ Design } & \multirow{3}{*}{ Sex } & \multicolumn{2}{|c|}{ Participants } & \multirow{3}{*}{ Dose or Placebo } & \multicolumn{2}{|c|}{ Intervention } & \multirow{3}{*}{ HMB n } & \multirow{3}{*}{$\begin{array}{c}\text { Control } \\
\mathrm{n}\end{array}$} & \multicolumn{4}{|c|}{ Outcome Measure } & \multirow{3}{*}{$\begin{array}{c}\text { Dietary } \\
\text { Assessment and } \\
\text { Protein Ingestion }\end{array}$} \\
\hline & & & & Age & Training Status & & $\begin{array}{l}\text { Duration } \\
\text { (Weeks) }\end{array}$ & Training & & & \multicolumn{2}{|c|}{ Strength } & \multicolumn{2}{|c|}{ Body Composition } & \\
\hline & & & & & & & & & & & $\begin{array}{l}\text { Upper } \\
\text { Body }\end{array}$ & $\begin{array}{l}\text { Lower } \\
\text { Body }\end{array}$ & Fat-free mass & $\begin{array}{l}\text { Fat } \\
\text { mass }\end{array}$ & \\
\hline $\begin{array}{l}\text { Asadi et al., } \\
2017 \text { [10] }\end{array}$ & Japan & $\begin{array}{l}\text { Randomized } \\
\text { controlled } \\
\text { trial (RCT) } \\
\text { Double } \\
\text { blinded (DB) }\end{array}$ & Male(M) & $21.4 \pm 0.7$ & Not Described & $\begin{array}{l}3 \mathrm{~g} \mathrm{HMB} \text {-FA } \\
\text { or polydextrose }\end{array}$ & 6 & $\begin{array}{l}2 \times / \mathrm{w} \\
3 \text { sets of } 8-12 \\
\text { repetitions per set } \\
\text { (rep) at } 75-85 \% \text { of } \\
1 \text { repetition } \\
\text { maximum (RM) }\end{array}$ & 8 & 8 & $\begin{array}{l}\text { Bench } \\
\text { press }\end{array}$ & Leg Press & - & - & $\begin{array}{l}\text { 3-day diet records } \\
\text { week } 0 \text { and week } 6 \\
\text { Protein ingestion } \\
\sim 1.45 \mathrm{~g} / \mathrm{kg} / \mathrm{d}\end{array}$ \\
\hline $\begin{array}{l}\text { Jakubowski } \\
\text { et al., 2019 [9] }\end{array}$ & Canada & $\begin{array}{l}\text { RCT } \\
\text { DB }\end{array}$ & M & $22.5 \pm 2.2$ & $\begin{array}{l}\text { Trained (TR) } \\
\text { (Recreationally } \\
\text { trained-2x/week) }\end{array}$ & $\begin{array}{l}3 \mathrm{~g} \mathrm{HMB}-\mathrm{Ca}+50 \mathrm{~g} \\
\text { Whey Protein or } \\
50 \mathrm{~g} \text { Whey Protein }\end{array}$ & 12 & $\begin{array}{l}3-5 \mathrm{x} / \mathrm{w} \\
\text { Phase } 1: 8 \mathrm{w} \\
\text { Undulating } \\
\text { periodized } \\
\text { resistance-training } \\
\text { (UPRT) } \\
\text { Phase 2: } 2 \mathrm{w} \\
\text { overreaching } \\
\text { Phase } 3: 2 \mathrm{w}\end{array}$ & 13 & 13 & $\begin{array}{l}\text { Bench } \\
\text { press }\end{array}$ & Squat & $\begin{array}{l}\text { Dual X-ray } \\
\text { absorptiometry } \\
\text { (DXA) }\end{array}$ & DXA & $\begin{array}{l}\text { 3-day diet records } \\
\text { weeks } 0,8 \text { and } 12 . \\
\text { Protein ingestion } \\
\sim 1.8-1.9 \mathrm{~g} / \mathrm{kg} / \mathrm{d}\end{array}$ \\
\hline $\begin{array}{l}\text { Kreider et al. } \\
\text { (2000) [5] }\end{array}$ & USA & $\begin{array}{l}\text { RCT } \\
\text { DB }\end{array}$ & M & $20.0 \pm 1.5$ & TR & $\begin{array}{l}3 \mathrm{~g} \mathrm{HMB}-\mathrm{Ca}, \\
99 \mathrm{~g} / \mathrm{d} \text { of glucose, } \\
1.1 \mathrm{~g} \mathrm{Na}_{2} \mathrm{HPO}_{4}, \\
1.2 \mathrm{~K}_{2} \mathrm{PO}_{4} \text { and } \\
3 \mathrm{~g} / \mathrm{d} \text { of Taurine or } \\
\text { a placebo without } \\
\mathrm{HMB}-\mathrm{Ca}\end{array}$ & 4 & $\begin{array}{l}4 \times / \text { week, } 1 \text { to } 3 \text { sets } \\
\text { of } 2-8 \text { rep, } 60 \text { to } \\
95 \% \text { of } 1 \mathrm{RM}(+3 \mathrm{x} \\
\text { week of agility } \\
\text { /sprint training) }\end{array}$ & 14 & 14 & $\begin{array}{l}\text { Bench } \\
\text { Press }\end{array}$ & Squat & DXA & DXA & $\begin{array}{l}\text { 4-day nutritional } \\
\text { intake assessment } \\
\text { day } 0 \text { and day } 28 \text {. } \\
\text { Protein ingestion } \\
1.5-2.4 \mathrm{~g} / \mathrm{kg} / \mathrm{d}\end{array}$ \\
\hline $\begin{array}{l}\text { Nissen et al., } \\
1996 \text { [2] }\end{array}$ & USA & $\begin{array}{l}\text { RCT } \\
\text { DB? } \\
\text { (not clear) }\end{array}$ & M & $19-29$ & $\begin{array}{l}\text { Untrained (UT) } \\
\text { (at least } \\
3 \text { months) }\end{array}$ & $\begin{array}{l}3 \mathrm{~g} \text { HMB-Ca }+ \\
\text { MET-Rx ( } 37 \mathrm{~g} \text { milk } \\
\text { protein) or } \\
\text { MET-Rx }\end{array}$ & 7 & $\begin{array}{l}3 \times / w \\
3 \text { sets of } 3-5 \text { rep at } \\
90 \% \text { of } 1 \text { RM. }\end{array}$ & 14 & 14 & $\begin{array}{l}\text { Several } \\
\text { upper } \\
\text { body } \\
\text { exercises }\end{array}$ & $\begin{array}{l}\text { Several } \\
\text { lower body } \\
\text { exercises }\end{array}$ & $\begin{array}{l}\text { Total body } \\
\text { electrical } \\
\text { conductivity } \\
\text { (TOBEC) }\end{array}$ & TOBEC & $\begin{array}{l}\text { No dietary } \\
\text { assessment for } \\
\text { study } 2 \text {. Estimated } \\
\text { protein intake } \\
\sim 1.8-2 \mathrm{~g} / \mathrm{kg} / \mathrm{d}\end{array}$ \\
\hline $\begin{array}{l}\text { Panton et al., } \\
2000[4]\end{array}$ & USA & $\begin{array}{l}\text { RCT } \\
\text { DB }\end{array}$ & M/Female(F) & $\begin{array}{l}25 \pm 1.2(\mathrm{M}) \\
23 \pm 0.6(\mathrm{~F})\end{array}$ & $\begin{array}{l}\mathrm{TR}(>6 \text { months } \\
\text { experience)/UT } \\
\text { (not training for } \\
\text { at least } \\
6 \text { months) } \\
\end{array}$ & $\begin{array}{l}3 \mathrm{~g} \text { HMB-Ca or } \\
\text { rice flour }\end{array}$ & 4 & $\begin{array}{l}3 \mathrm{x} / \mathrm{w} \text {. 3-6 rep } 90 \% \\
\text { 1RM. }\end{array}$ & $\begin{array}{l}39 \\
(21 \mathrm{M} / 18 \mathrm{~F})\end{array}$ & $\begin{array}{l}36 \\
(18 \mathrm{M} / 18 \mathrm{~F})\end{array}$ & $\begin{array}{l}\text { Bench } \\
\text { press }\end{array}$ & $\begin{array}{l}\text { Leg Press } \\
\text { (M) } \\
\text { Leg } \\
\text { Extension } \\
\text { (F) } \\
\end{array}$ & UWW & UWW & Not described \\
\hline $\begin{array}{l}\text { Slater et al. } \\
\text { (2001) [6] }\end{array}$ & Australia & $\begin{array}{l}\text { RCT } \\
\text { DB }\end{array}$ & M & $24.5 \pm 1.7$ & TR & $\begin{array}{l}3 \mathrm{~g} \mathrm{HMB} \text {-Ca } \\
\text { (Standard } \\
\text { encapsulation vs. } \\
\text { Time Release) or } \\
\text { rice flour }\end{array}$ & 6 & $\begin{array}{l}2-3 \times / w .4-6 \\
\text { repetitions for } \\
3-5 \text { sets ( } 24 \text { to } 32 \text { sets } \\
\text { per session) }\end{array}$ & 7 & 7 & $\begin{array}{l}\text { Bench } \\
\text { press }\end{array}$ & Leg Press & DXA & DXA & $\begin{array}{l}\text { Regularly dietary } \\
\text { logs } \\
\text { Pre } 1.7 \mathrm{~g} / \mathrm{kg} / \mathrm{d} \\
\text { Post } 2.4 \mathrm{~g} / \mathrm{kg} / \mathrm{d}\end{array}$ \\
\hline
\end{tabular}


Table 2. Cont.

\begin{tabular}{|c|c|c|c|c|c|c|c|c|c|c|c|c|c|c|c|}
\hline \multirow{3}{*}{ Study } & \multirow{3}{*}{ Country } & \multirow[t]{3}{*}{ Design } & \multicolumn{3}{|c|}{ Participants } & \multicolumn{4}{|c|}{ Intervention } & & \multicolumn{4}{|c|}{ Outcome Measure } & \multirow{3}{*}{$\begin{array}{c}\text { Dietary } \\
\text { Assessment and } \\
\text { Protein Ingestion }\end{array}$} \\
\hline & & & \multirow[t]{2}{*}{ Sex } & \multirow[t]{2}{*}{ Age } & \multirow[t]{2}{*}{ Training Status } & \multirow[t]{2}{*}{ Dose or Placebo } & \multirow{2}{*}{$\begin{array}{l}\text { Duration } \\
\text { (Weeks) }\end{array}$} & \multirow[t]{2}{*}{ Training } & \multirow[t]{2}{*}{ HMB n } & \multirow{2}{*}{$\begin{array}{c}\text { Control } \\
n\end{array}$} & \multicolumn{2}{|c|}{ Strength } & \multicolumn{2}{|c|}{ Body Composition } & \\
\hline & & & & & & & & & & & $\begin{array}{l}\text { Upper } \\
\text { Body }\end{array}$ & $\begin{array}{l}\text { Lower } \\
\text { Body }\end{array}$ & Fat-free mass & $\begin{array}{c}\text { Fat } \\
\text { mass }\end{array}$ & \\
\hline $\begin{array}{l}\text { Stahn et al. } \\
\text { (2020) [39] }\end{array}$ & USA & $\begin{array}{l}\text { RCT } \\
\text { DB }\end{array}$ & M & $22.1 \pm 1.5$ & $\begin{array}{l}\text { UT (for the past } \\
6 \text { months) }\end{array}$ & $\begin{array}{l}3 \mathrm{~g} \mathrm{HMB}-\mathrm{Ca}+30 \mathrm{~g} \\
\text { Whey Protein } \\
\text { (daily). }+30 \mathrm{~g} \\
\text { carbohydrate } \\
\text { supplement only } \\
\text { on training days or } \\
\text { the supplements + } \\
\text { microcrystalline } \\
\text { cellulose as } \\
\text { placebo }\end{array}$ & 12 & $\begin{array}{l}\text { 4x/w } \\
\text { upper/lower body } \\
\text { split routine. } \\
\text { Weeks 1-6: linear } \\
\text { periodization. Week } \\
7 \text { tapering. Weeks } \\
\text { 8-12: undulating } \\
\text { periodization }\end{array}$ & 8 & 7 & $\begin{array}{l}\text { Bench } \\
\text { Press }\end{array}$ & Leg Press & $\begin{array}{l}\text { Bioelectrical } \\
\text { impedance } \\
\text { (BIA) }\end{array}$ & BIA & $\begin{array}{l}\text { No dietary } \\
\text { assessment }\end{array}$ \\
\hline $\begin{array}{l}\text { Teixeira et al., } \\
\text { 2019a [8] }\end{array}$ & Portugal & $\begin{array}{l}\text { RCT } \\
\text { DB }\end{array}$ & $\mathrm{M}$ & $31.7 \pm 7.6$ & $\begin{array}{l}\text { TR (>1 year } \\
\text { experience) }\end{array}$ & $\begin{array}{l}3 \mathrm{~g} \text { HMB-Ca or } 3 \mathrm{~g} \\
\text { HMB-FA or Mg } \\
\text { stearate as placebo }\end{array}$ & 8 & $\begin{array}{l}3 \mathrm{x} / \mathrm{w} \\
\text { Weeks 1-3, 3-4 sets } \\
\text { 12RM } \\
\text { Weeks } 4-6,3-4 \text { sets } \\
\text { 10RM } \\
\text { Weeks 7-8, } 4 \text { sets } \\
\text { 8RM }\end{array}$ & 20 & 10 & - & - & DXA & DXA & $\begin{array}{l}\text { 3-day dietary logs } \\
\text { weeks } 0,4 \text { and } 8 . \\
\text { Dietary } \\
\text { instructions to } \\
\text { adjust energy and } \\
\text { protein ingestion. } \\
\text { Protein ingestion } \\
\text { 3.0-3.3 } \mathrm{g} / \mathrm{kg} / \mathrm{d}\end{array}$ \\
\hline $\begin{array}{l}\text { Teixeira et al., } \\
\text { 2019b [12] }\end{array}$ & Portugal & $\begin{array}{l}\text { RCT } \\
\text { DB }\end{array}$ & $\mathrm{M}$ & $31.7 \pm 7.6$ & $\begin{array}{l}\text { TR (>1 year } \\
\text { experience) }\end{array}$ & $\begin{array}{l}3 \mathrm{~g} \text { HMB-Ca or } 3 \mathrm{~g} \\
\text { HMB-FA or Mg } \\
\text { stearate as placebo }\end{array}$ & 8 & $\begin{array}{l}3 \mathrm{x} / \mathrm{w} \\
\text { Weeks 1-3, 3-4 sets } \\
\text { 12RM } \\
\text { Weeks } 4-6,3-4 \text { sets } \\
\text { 10RM } \\
\text { Weeks 7-8, } 4 \text { sets } \\
\text { 8RM }\end{array}$ & 20 & 10 & $\begin{array}{l}\text { Bench } \\
\text { Press }\end{array}$ & Squat & - & - & $\begin{array}{l}\text { 3-day dietary logs } \\
\text { weeks } 0,4 \text { and } 8 \text {. } \\
\text { Dietary } \\
\text { counselling to } \\
\text { adjust energy and } \\
\text { protein ingestion } \\
\text { Protein ingestion } \\
\text { 3.0-3.3 } \mathrm{g} / \mathrm{kg} / \mathrm{d}\end{array}$ \\
\hline $\begin{array}{l}\text { Thomson } \\
\text { et al., 2009 } \\
\text { [37] }\end{array}$ & $\begin{array}{l}\text { New } \\
\text { Zealand }\end{array}$ & $\begin{array}{l}\text { RCT } \\
\text { DB }\end{array}$ & $\mathrm{M}$ & $24 \pm 4$ & $\begin{array}{l}\text { TR (>3 years } \\
\text { experience) }\end{array}$ & $\begin{array}{l}3 \mathrm{~g} \text { HMB-Ca or } \\
\text { corn starch }\end{array}$ & 9 & $3 \mathrm{x} / \mathrm{w}$ & 13 & 9 & $\begin{array}{l}\text { Bench } \\
\text { press }\end{array}$ & $\begin{array}{l}\text { Leg } \\
\text { extension }\end{array}$ & BIA & BIA & $\begin{array}{l}\text { Diets evaluated by } \\
\text { 3-day records at } \\
\text { weeks } 0 \text { and } 9 . \\
\text { Protein ingestion: } \\
\text { not stated. }\end{array}$ \\
\hline $\begin{array}{l}\text { Tritto et al., } \\
2019 \text { [38] }\end{array}$ & Brazil & $\begin{array}{l}\text { RCT } \\
\text { DB }\end{array}$ & $\mathrm{M}$ & $25.3 \pm 3.7$ & $\begin{array}{l}\mathrm{TR}(>6 \text { months } \\
\text { experience) }\end{array}$ & $\begin{array}{l}3 \mathrm{~g} \text { HMB-Ca or } 3 \mathrm{~g} \\
\text { HMB-FA or } \\
\text { corn starch }\end{array}$ & 12 & $\begin{array}{l}2 \times / w \\
3-4 \text { sets } 8-10 \text { RM }\end{array}$ & 29 & 15 & $\begin{array}{l}\text { Bench } \\
\text { press }\end{array}$ & Leg Press & DXA & DXA & $\begin{array}{l}\text { Diets evaluated by } \\
\text { 3-day records at } \\
\text { weeks } 0 \text { and } 12 \text {. } \\
\text { Protein ingestion: } \\
1.9-2.1 \mathrm{~g} / \mathrm{kg} / \mathrm{d} \text {. }\end{array}$ \\
\hline
\end{tabular}

M: Male; F: Female; BIA: Bioelectrical impedance; DB: Double blinded; DXA: Dual X-ray absorptiometry; FFM: Fat-free mass; FM: Fat mass; RCT: Randomized controlled trial; rep: repetitions per set; RM; Repetition maximum; TOBEC: Total body electrical conductivity; TR: Trained; UPRT: Undulating periodized resistance-training; UT: Untrained; UWW: Underwater weighting. 


\subsection{Body Mass and Composition}

Total body mass was evaluated in eleven trials. The mean TBM gain in HMB-supplemented subjects was $1.12 \mathrm{~kg} \pm 1.89 \mathrm{~kg}$ versus $0.78 \mathrm{~kg} \pm 1.31 \mathrm{~kg}$ gain in the placebo group. The mean difference between placebo and experimental was $0.34 \mathrm{~kg}(95 \%$ CI $0.03,0.66, p<0.05)$. Eleven trials measured lean body mass changes. Mean difference between supplemented and placebo groups was $0.29 \mathrm{~kg}$ (95\% CI $-0.01,0.60, p=0.06)$. The HMB-supplemented groups gained an average of $1.57 \mathrm{~kg} \pm 1.75 \mathrm{~kg}$ and the placebo groups gained $1.17 \mathrm{~kg} \pm 1.45 \mathrm{~kg}$ of FFM. Mean fat loss was equivalent in these same studies. HMB-supplemented group lost $0.73 \mathrm{~kg} \pm 1.68 \mathrm{~kg}$, and the placebo groups lost $0.47 \mathrm{~kg} \pm 1.43 \mathrm{~kg}$. The mean difference between groups was $0.10 \mathrm{~kg}(95 \% \mathrm{CI}-0.42,0.23, p=0.57)$ (Figure 2).



Figure 2. Forest plot of a random-effects meta-analysis for the effect of HMB supplementation on changes in TBM (9 studies, 10 trials), lean body mass (9 studies, 10 trials) and fat mass (10 studies, 9 trials). Results are presented as mean difference between HMB supplemented (Experimental) and Placebo group with 95\% CIs. HMB, $\beta$-hydroxy- $\beta$-methylbutyrate; CIs, Confidence intervals.

\subsection{Gains in Muscle Strength}

Strength was an investigated outcome in 8 of the 11 analyzed studies. In these studies, subjects that undertook RET and placebo increased their total 1RM strength by $30.6 \mathrm{~kg} \pm 29.9 \mathrm{~kg}$. HMB-supplemented individuals increased their total 1RM strength by $32.0 \mathrm{~kg} \pm 31.4 \mathrm{~kg}$. Mean difference between groups 
in total 1RM strength was $1.11 \mathrm{~kg}(95 \% \mathrm{CI}-1.90,4.12)$, which was not statistically significant $(p=0.47)$. Total 1RM strength was based on bench press and lower body 1RM data. Bench press 1RM strength gains were equivalent to $9.5 \mathrm{~kg} \pm 4.6 \mathrm{~kg}$ in studies testing HMB supplemented subjects. In the same studies, the placebo group increased its strength by an average of $9.6 \mathrm{~kg} \pm 4.9 \mathrm{~kg}$. The mean difference between groups in bench press $1 \mathrm{RM}$ strength was not statistically significant $(0.52 \mathrm{~kg}(95 \% \mathrm{CI}$ $-1.72,1.75, p=0.41)$ ). Finally, lower body 1 RM strength was also not significantly affected by HMB supplementation. The mean lower body $1 \mathrm{RM}$ difference between experimental and placebo groups was $2.82 \mathrm{~kg}(95 \% \mathrm{CI}:-2.37,8.00 p=0.29)$ (Figure 3).

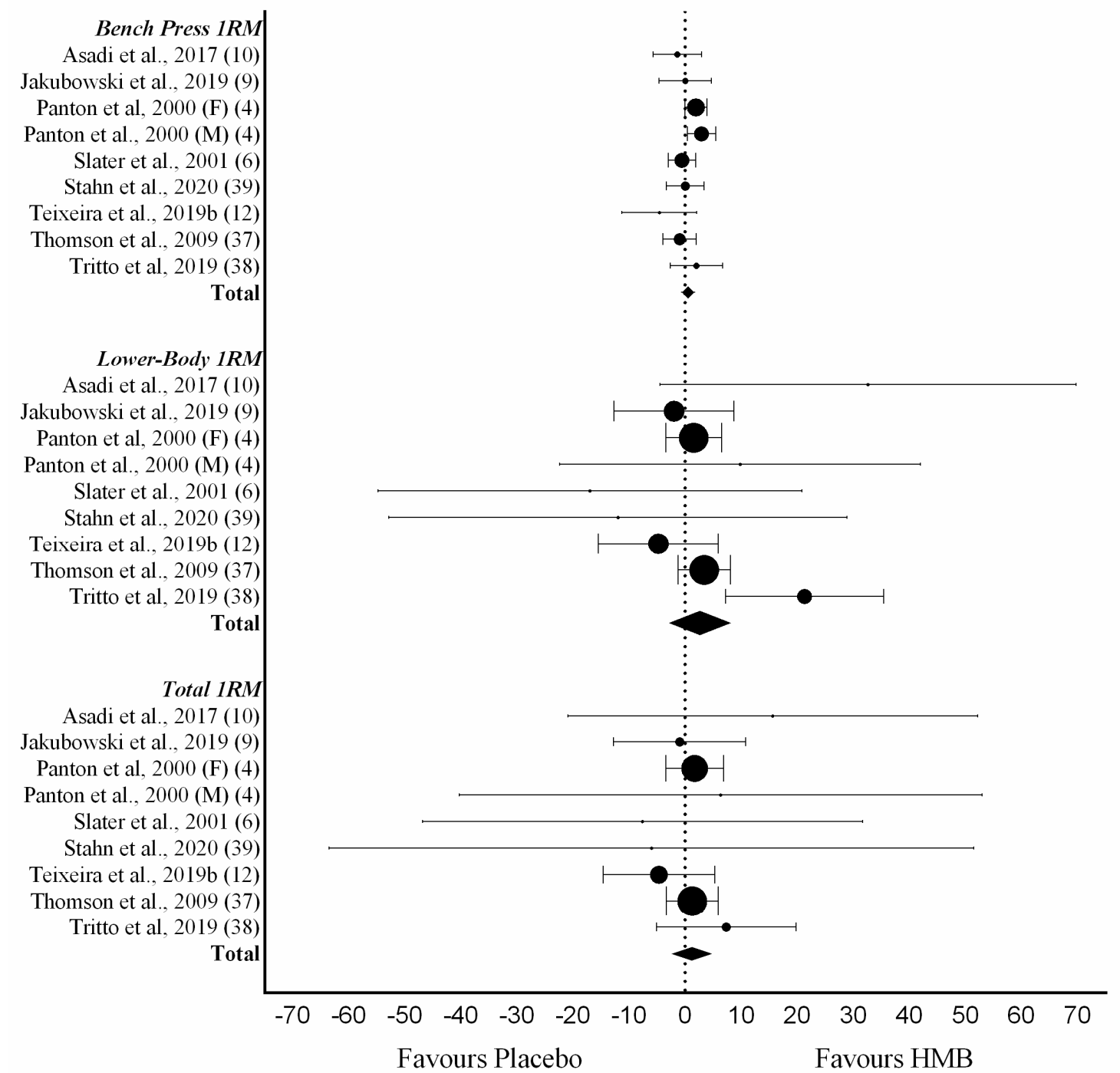

Figure 3. Forest plot of a random-effects meta-analysis for the effect of HMB supplementation on changes in strength. Eight studies (nine trials) were analyzed for bench press 1RM, lower body $1 \mathrm{RM}(\mathrm{kg})$ and total 1RM. Results are presented as mean difference between HMB supplemented (Experimental) and Placebo group with 95\% CIs. HMB, $\beta$-hydroxy- $\beta$-methylbutyrate; CIs, Confidence intervals.

\subsection{Sensitivity Analyses}

All outcomes were submitted to sensitivity analysis using the "remove- 1 " strategy. The outcome "total body mass" was significantly influenced by the removal of 1 study. Studies were removed according to the criteria of 3 or more domains judged as high or unclear risk of bias. When the results of Nissen et al., [2] were removed from the meta-analysis, the mean difference between treatments 
turned out to be non-significant $(0.20 \mathrm{~kg}(95 \% \mathrm{CI}-0.13,0.54, p=0.20))$. Therefore, the main effect of HMB supplementation in TMB, presented in Figure 2 should be interpreted with caution.

\section{Discussion}

This systematic review and meta-analysis showed that HMB ingestion does not augment RET-induced changes in body composition or strength following weeks or RET (7.6 $\pm 4.0 \mathrm{wk})$ in adults between the ages of 18 and 45 years old. These findings are in line with results from previous meta-analyses focusing on HMB supplementation $[7,11,28]$. Nevertheless, a small significant effect was observed for TBM. However, sensitivity analyses revealed that this result was strongly influenced by one study that scored as "high risk" in three domains of the risk of bias analysis [2]. This highlights the importance of a qualitative view when interpreting the results of a meta-analysis.

A notable and relevant facet of the present meta-analysis is that our conclusions were based on studies conducted in non-athletes. Although, this was not one of the main objectives of the present meta-analysis when designing inclusion and exclusion criteria, our conclusions derived mainly from studies in which untrained persons were studied (Table 2). This is important because two recent meta-analyses $[11,28]$ showed no substantive effects of ingesting HMB when combined with resistance exercise, but on studies conducted mostly in athletes. Therefore, it seems reasonable to conclude that our data, viewed collectively with previous work $[11,28]$, show that HMB is not a valuable nutritional supplement to be consumed by athletes or non-athletes aiming to improve resistance exercise-induced gains in FFM and strength.

$\mathrm{HMB}$, as would be expected as a metabolite of leucine, can acutely activate skeletal muscle protein synthesis and the main signaling pathways leading to protein synthesis $[15,16]$. Increased muscle protein synthesis is a requisite for promoting a positive net muscle protein balance and increasing muscle mass as a result of RET [40]. Some studies have reported extraordinarily greater lean mass and strength gains by individuals ingesting HMB [35,36], or HMB + ATP [41] when undertaking RET. It is relevant to highlight that Kraemer et al. [35], and Wilson et al., [36] met the inclusion criteria for this meta-analysis but were excluded due to the marked asymmetry that inclusion of those studies created, as seen in the funnel plot analyses when the data were present (Supplementary Figure S1). Kraemer et al. [35], reported $\sim 9.3 \mathrm{~kg}$ gain of FFM, and Wilson et al. [36] reported $7.4 \mathrm{~kg}$ of FFM gain over 12 weeks of resistance exercise and HMB supplementation. However, FFM gains of such magnitude are similar to those reported by the subjects submitted to resistance exercise and in the use of androgen anabolic steroids [42]. Also, these same studies [35,36] reported high FM loss (i.e., $>5 \mathrm{~kg}$ ) in the groups ingesting HMB. Nevertheless, the present meta-analysis found that average FM loss is $0.73 \mathrm{~kg} \pm 1.68 \mathrm{~kg}$ in trials testing HMB during RET. Hence, it is reasonable, as we have stressed previously [9], to exclude studies that report seemingly unparalleled results (i.e., $>7 \mathrm{~kg}$ in FFM) in 12 weeks of RET.

There are no studies measuring the acute protein synthetic response to a protein meal/supplement or to a resistance exercise bout in the presence of HMB. Therefore, it is not known if the acute effects of $\mathrm{HMB}$ on protein synthesis $[15,16]$ are additive to the expected response to the ingestion of proteins or a resistance exercise bout, but it would seem unlikely so long as leucine was ingested at a sufficient dose [15]. Nevertheless, some studies [2,3,9,39], including one from our group [9], have tested the long-term effects of ingesting $\mathrm{HMB}$ together with high-quality protein on the adaptation to resistance exercise. Two of these studies [3,9], showed no significant benefit to body composition changes or strength gains caused by RET of adding HMB to protein-containing nutritional supplements, $75 \mathrm{~g}$ and $50 \mathrm{~g}$ respectively $[3,9]$. In addition, Shirato et al. [43] were not able to find any significant effect of adding only HMB ( $3 \mathrm{~g}$ ) to a $\sim 37 \mathrm{~g} /$ day of whey protein supplement on the recovery from eccentric exercise. This is relevant because one of the main statements used during the prescription of HMB is based on its potential effects on accelerating skeletal muscle recovery after resistance exercise sessions [24]. In contrast, Nissen et al. [2] showed a higher increase in lean body mass and strength when HMB was added to a nutritional supplement containing $37 \mathrm{~g}$ of milk protein. Also, Stahn et al. [39] showed that ingesting $3 \mathrm{~g}$ of HMB-Ca added to $30 \mathrm{~g}$ of whey protein was able to increase fat-free mass in young men. 
However, the placebo group in Stahn et al. [39] (ingesting $30 \mathrm{~g}$ whey protein only) did not actually show a significant increase in whole-body FFM. Therefore, the conclusions of that study [39] are perplexing, since it would be expected that 12 weeks of RET would result in a significant increase in whole-body FFM even in the placebo group $[9,44]$. Nevertheless, the fact that HMB is not effective in producing significant effects in young subjects performing resistance exercise does not exclude the possibility of potential effects in other populations or physiological states. In fact, a recent meta-analysis revealed a small significant effect for HMB supplementation on increasing muscle mass and function in a variety of clinical conditions characterized by loss of skeletal muscle mass and weakness [45]. However, older subjects performing physical activity seem not to have further benefits on physical function by ingesting HMB when compared to performing physical activity only [46]. Finally, the ingestion of HMB by subjects performing non-resistance exercise activities might also produce distinct results when compared to our conclusions since a few trials have shown significant changes in body composition and performance variables as an effect of HMB supplementation [47,48].

One important limitation of this meta-analysis is the number of studies fitting inclusion/exclusion criteria and remaining after screening by the risk of bias analysis. Eleven studies might be considered a small number when drawing final conclusions. However, this analysis is in line with some of the recently published meta-analyses studying HMB [11,28]. In addition, although there was no significant heterogeneity observed in the analysis of strength gains, the variability between RET protocols might be another limiting factor. However, 8 out of 11 selected studies used RET protocols with a training frequency of 3 days/week or more. Additionally, six studies utilized RET programs of at least 8 weeks (Table 2). Protein ingestion was also cited as a potential limiting factor influencing resistance exercise responses in HMB-supplemented individuals [28]. Holland et al. [28] postulated that HMB might not enhance RET gains in individuals already ingesting $>1.6 \mathrm{~g}$ of protein/day. Still, only two of the seven selected studies by their meta-analysis reported daily protein ingestion of $>1.6 \mathrm{~g}$ of protein/day and additional four studies did not report protein ingestion. Hence, in our view any statement related to such protein-dependence on daily protein ingestion is tenuous. In our meta-analysis, 6 out of 11 studies reported protein ingestions in the range of $1.9-3.3 \mathrm{~g} / \mathrm{kg} / \mathrm{day}$, and one additional study reported daily protein ingestion of $1.45 \mathrm{~g} / \mathrm{kg}$. Also, three studies did not state the protein ingestion. Based on such data, it is not possible to perform any analysis as to what protein intake might be needed to see an effect of HMB supplementation. We would posit, however, that dietary protein, even though its impact on hypertrophy is relatively small [44], would be far more potent than a single leucine metabolite.

\section{Conclusions}

This systematic review and meta-analysis showed that HMB supplementation during RET may result in a small increase in TBM but does not result in a significant enhancement of gains in FFM or losses of FM. Thus, there is no rationale for prescription of HMB as a supplement to improve body composition caused by RET in young subjects. In addition, effects on strength were also not significant. Furthermore, risk of bias and sensitivity analysis suggest that some studies reporting significant HMB effects to optimize RET adaptations are not commonly reproducible $[35,36]$ or have a considerable risk of bias [2]. Our results, particularly when viewed in light of other meta-analyses reaching similar conclusions $[11,28]$, show that HMB is not an effective anabolic supplement.

Supplementary Materials: The following are available online at http://www.mdpi.com/2072-6643/12/5/1523/s1: Figure S1: Funnel plots showing relation between mean differences (MD) in x axis and standard errors (SE) for mean differences. Figure S2: Risk of bias summary for selected studies. Table S1: Characteristics of the studies eligible for inclusion. Table S2: List of studies removed from the meta-analysis after data collection and asymmetry with reason for exclusion. File S1-Search Strategy.

Author Contributions: Conceptualization: E.A.N., F.J.T., J.S.J., R.W.M., and S.M.P.; Investigation: E.A.N., F.J.T., J.S.J., L.B., R.W.M., and V.V.; Formal analysis: E.A.N. and J.S.J.; Project administration: E.A.N., J.S.J., and S.M.P.; Resources: S.M.P.; Supervision: S.M.P.; Writing-original draft: E.A.N. and S.M.P.; Writing-review, editing, and approval of final version: All authors. All authors have read and agreed to the published version of the manuscript. 
Funding: This research received no external funding, EAN is a Tier 2 Productivity Fellow for the Brazilian National Council for Scientific and Technological Development (CNPq), grant number 308584/2019-8.

Conflicts of Interest: The authors declare no conflict of interest.

\section{References}

1. Van Koevering, M.; Nissen, S. Oxidation of leucine and alpha-ketoisocaproate to beta-hydroxy-betamethylbutyrate in vivo. Am. J. Physiol. 1992, 262, E27-E31. [CrossRef] [PubMed]

2. Nissen, S.; Sharp, R.; Ray, M.; Rathmacher, J.A.; Rice, D.; Fuller, J.C., Jr.; Connelly, A.S.; Abumrad, N. Effect of leucine metabolite beta-hydroxy-beta-methylbutyrate on muscle metabolism during resistance-exercise training. J. Appl. Physiol. 1996, 81, 2095-2104. [CrossRef] [PubMed]

3. Kreider, R.B.; Ferreira, M.; Wilson, M.; Almada, A.L. Effects of calcium beta-hydroxy-beta-methylbutyrate (HMB) supplementation during resistance-training on markers of catabolism, body composition and strength. Int. J. Sports Med. 1999, 20, 503-509. [CrossRef] [PubMed]

4. Panton, L.B.; Rathmacher, J.A.; Baier, S.; Nissen, S. Nutritional supplementation of the leucine metabolite beta-hydroxy-beta-methylbutyrate (hmb) during resistance training. Nutrition 2000, 16, 734-739. [CrossRef]

5. Kreider, R.B.; Ferreira, M.; Greenwood, M.; Wilson, M.; Grindstaff, P.; Plisk, J.; Reinardy, J.; Cantler, E.; Almada, A.L. Effects of Calcium $\beta$-HMB Supplementation During Training on Markers of Catabolism, Body Composition, Strength and Sprint Performance. J. Exerc. Physiol. Online 2000, 3, 11.

6. Slater, G.; Jenkins, D.; Logan, P.; Lee, H.; Vukovich, M.; Rathmacher, J.A.; Hahn, A.G. Beta-hydroxybeta-methylbutyrate $(\mathrm{HMB})$ supplementation does not affect changes in strength or body composition during resistance training in trained men. Int. J. Sport Nutr. Exerc. Metab. 2001, 11, 384-396. [CrossRef]

7. Rowlands, D.S.; Thomson, J.S. Effects of beta-hydroxy-beta-methylbutyrate supplementation during resistance training on strength, body composition, and muscle damage in trained and untrained young men: A meta-analysis. J. Strength Cond. Res. 2009, 23, 836-846. [CrossRef]

8. Teixeira, F.J.; Matias, C.N.; Monteiro, C.P.; Valamatos, M.J.; Reis, J.F.; Batista, A.; Oliveira, A.C.; Alves, F.; Sardinha, L.B.; Phillips, S.M. No effect of HMB or alpha-HICA supplementation on training-induced changes in body composition. Eur. J. Sport Sci. 2019, 19, 802-810. [CrossRef]

9. Jakubowski, J.S.; Wong, E.P.T.; Nunes, E.A.; Noguchi, K.S.; Vandeweerd, J.K.; Murphy, K.T.; Morton, R.W.; McGlory, C.; Phillips, S.M. Equivalent Hypertrophy and Strength Gains in beta-Hydroxy-beta-Methylbutyrateor Leucine-supplemented Men. Med. Sci. Sports Exerc. 2019, 51, 65-74. [CrossRef]

10. Asadi, A.; Arazi, H.; Suzuki, K. Effects of beta-Hydroxy-beta-methylbutyrate-free Acid Supplementation on Strength, Power and Hormonal Adaptations Following Resistance Training. Nutrients 2017, 9, 1316. [CrossRef]

11. Sanchez-Martinez, J.; Santos-Lozano, A.; Garcia-Hermoso, A.; Sadarangani, K.P.; Cristi-Montero, C. Effects of beta-hydroxy-beta-methylbutyrate supplementation on strength and body composition in trained and competitive athletes: A meta-analysis of randomized controlled trials. J. Sci. Med. Sport 2018, 21, 727-735. [CrossRef] [PubMed]

12. Teixeira, F.J.; Matias, C.N.; Monteiro, C.P.; Valamatos, M.J.; Reis, J.F.; Tavares, F.; Batista, A.; Domingos, C.; Alves, F.; Sardinha, L.B.; et al. Leucine Metabolites Do Not Enhance Training-induced Performance or Muscle Thickness. Med. Sci. Sports Exerc. 2019, 51, 56-64. [CrossRef] [PubMed]

13. Pimentel, G.D.; Rosa, J.C.; Lira, F.S.; Zanchi, N.E.; Ropelle, E.R.; Oyama, L.M.; Oller do Nascimento, C.M.; de Mello, M.T.; Tufik, S.; Santos, R.V. Beta-Hydroxy-beta-methylbutyrate (HMbeta) supplementation stimulates skeletal muscle hypertrophy in rats via the mTOR pathway. Nutr. Metab. (Lond.) 2011, 8, 11. [CrossRef] [PubMed]

14. Pinheiro, C.H.; Gerlinger-Romero, F.; Guimaraes-Ferreira, L.; de Souza, A.L., Jr.; Vitzel, K.F.; Nachbar, R.T.; Nunes, M.T.; Curi, R. Metabolic and functional effects of beta-hydroxy-beta-methylbutyrate (HMB) supplementation in skeletal muscle. Eur. J. Appl. Physiol. 2012, 112, 2531-2537. [CrossRef]

15. Wilkinson, D.J.; Hossain, T.; Hill, D.S.; Phillips, B.E.; Crossland, H.; Williams, J.; Loughna, P.; Churchward-Venne, T.A.; Breen, L.; Phillips, S.M.; et al. Effects of leucine and its metabolite beta-hydroxy-beta-methylbutyrate on human skeletal muscle protein metabolism. J. Physiol. 2013, 591, 2911-2923. [CrossRef] 
16. Wilkinson, D.J.; Hossain, T.; Limb, M.C.; Phillips, B.E.; Lund, J.; Williams, J.P.; Brook, M.S.; Cegielski, J.; Philp, A.; Ashcroft, S.; et al. Impact of the calcium form of beta-hydroxy-beta-methylbutyrate upon human skeletal muscle protein metabolism. Clin. Nutr. 2018, 37, 2068-2075. [CrossRef]

17. Giron, M.D.; Vilchez, J.D.; Salto, R.; Manzano, M.; Sevillano, N.; Campos, N.; Argiles, J.M.; Rueda, R.; Lopez-Pedrosa, J.M. Conversion of leucine to beta-hydroxy-beta-methylbutyrate by alpha-keto isocaproate dioxygenase is required for a potent stimulation of protein synthesis in L6 rat myotubes. J. Cachexia Sarcopenia Muscle 2016, 7, 68-78. [CrossRef]

18. Kimura, K.; Cheng, X.W.; Inoue, A.; Hu, L.; Koike, T.; Kuzuya, M. Beta-Hydroxy-beta-methylbutyrate facilitates PI3K/Akt-dependent mammalian target of rapamycin and FoxO1/3a phosphorylations and alleviates tumor necrosis factor alpha/interferon gamma-induced MuRF-1 expression in C2C12 cells. Nutr. Res. 2014, 34, 368-374. [CrossRef]

19. Gerlinger-Romero, F.; Guimaraes-Ferreira, L.; Yonamine, C.Y.; Salgueiro, R.B.; Nunes, M.T. Effects of beta-hydroxy-beta-methylbutyrate (HMB) on the expression of ubiquitin ligases, protein synthesis pathways and contractile function in extensor digitorum longus (EDL) of fed and fasting rats. J. Physiol. Sci. 2018, 68, 165-174. [CrossRef]

20. Zanchi, N.E.; Gerlinger-Romero, F.; Guimaraes-Ferreira, L.; de Siqueira Filho, M.A.; Felitti, V.; Lira, F.S.; Seelaender, M.; Lancha, A.H., Jr. HMB supplementation: Clinical and athletic performance-related effects and mechanisms of action. Amino Acids 2011, 40, 1015-1025. [CrossRef]

21. Holecek, M. Beta-hydroxy-beta-methylbutyrate supplementation and skeletal muscle in healthy and muscle-wasting conditions. J. Cachexia Sarcopenia Muscle 2017, 8, 529-541. [CrossRef] [PubMed]

22. Gepner, Y.; Varanoske, A.N.; Boffey, D.; Hoffman, J.R. Benefits of beta-hydroxy-beta-methylbutyrate supplementation in trained and untrained individuals. Res. Sports Med. 2019, 27, 204-218. [CrossRef] [PubMed]

23. Portal, S.; Eliakim, A.; Nemet, D.; Halevy, O.; Zadik, Z. Effect of HMB supplementation on body composition, fitness, hormonal profile and muscle damage indices. J. Pediatr. Endocrinol. Metab. 2010, 23, 641-650. [CrossRef] [PubMed]

24. Silva, V.R.; Belozo, F.L.; Micheletti, T.O.; Conrado, M.; Stout, J.R.; Pimentel, G.D.; Gonzalez, A.M. Beta-hydroxy-beta-methylbutyrate free acid supplementation may improve recovery and muscle adaptations after resistance training: A systematic review. Nutr. Res. 2017, 45, 1-9. [CrossRef] [PubMed]

25. Fernandez-Landa, J.; Calleja-Gonzalez, J.; Leon-Guereno, P.; Caballero-Garcia, A.; Cordova, A.; Mielgo-Ayuso, J. Effect of the Combination of Creatine Monohydrate Plus HMB Supplementation on Sports Performance, Body Composition, Markers of Muscle Damage and Hormone Status: A Systematic Review. Nutrients 2019, 11, 2528. [CrossRef]

26. Chilibeck, P.D.; Kaviani, M.; Candow, D.G.; Zello, G.A. Effect of creatine supplementation during resistance training on lean tissue mass and muscular strength in older adults: A meta-analysis. Open Access J. Sports Med. 2017, 8, 213-226. [CrossRef]

27. Nissen, S.L.; Sharp, R.L. Effect of dietary supplements on lean mass and strength gains with resistance exercise: A meta-analysis. J. Appl. Physiol. (1985) 2003, 94, 651-659. [CrossRef]

28. Holland, B.M.; Roberts, B.M.; Krieger, J.W.; Schoenfeld, B.J. Does HMB Enhance Body Composition in Athletes? A Systematic Review and Meta-analysis. J. Strength Cond. Res. 2019, 20, 1-8. [CrossRef]

29. Fuller, J.C.; Sharp, R.L.; Angus, H.F.; Khoo, P.Y.; Rathmacher, J.A. Comparison of availability and plasma clearance rates of beta-hydroxy-beta-methylbutyrate delivery in the free acid and calcium salt forms. Br. J. Nutr. 2015, 114, 1403-1409. [CrossRef]

30. Higgins, J.P.T.; Thomas, J.; Chandler, J.; Cumpston, M.; Li, T.; Page, M.J.; Welch, V.A. (Eds.) Cochrane Handbook for Systematic Reviews of Interventions version 6.0 (updated July 2019); Cochrane: London, UK, 2019; Available online: www.training.cochrane.org/handbook (accessed on 1 February 2020).

31. Liberati, A.; Altman, D.G.; Tetzlaff, J.; Mulrow, C.; Gotzsche, P.C.; Ioannidis, J.P.; Clarke, M.; Devereaux, P.J.; Kleijnen, J.; Moher, D. The PRISMA statement for reporting systematic reviews and meta-analyses of studies that evaluate health care interventions: Explanation and elaboration. J. Clin. Epidemiol. 2009, 62, e1-e34. [CrossRef] 
32. Sterne, J.A.; Sutton, A.J.; Ioannidis, J.P.; Terrin, N.; Jones, D.R.; Lau, J.; Carpenter, J.; Rucker, G.; Harbord, R.M.; Schmid, C.H.; et al. Recommendations for examining and interpreting funnel plot asymmetry in meta-analyses of randomised controlled trials. BMJ 2011, 343, d4002. [CrossRef] [PubMed]

33. Schünemann, H.; Brozek, J.; Guyatt, G.; Oxman, A. GRADE Handbook for Grading Quality of Evidence and Strength of Recommendations. 2013; Available online: https://raining.cochrane.org/resource/gradehandbook (accessed on 1 February 2020).

34. The Nordic Cochrane Centre. RevMan Review Manager (RevMan) Version 5.3; The Nordic Cochrane Centre, The Cochrane Collaboration: Copenhagen, Denmark, 2014.

35. Kraemer, W.J.; Hatfield, D.L.; Volek, J.S.; Fragala, M.S.; Vingren, J.L.; Anderson, J.M.; Spiering, B.A.; Thomas, G.A.; Ho, J.Y.; Quann, E.E.; et al. Effects of amino acids supplement on physiological adaptations to resistance training. Med. Sci. Sports Exerc. 2009, 41, 1111-1121. [CrossRef] [PubMed]

36. Wilson, J.M.; Lowery, R.P.; Joy, J.M.; Andersen, J.C.; Wilson, S.M.; Stout, J.R.; Duncan, N.; Fuller, J.C.; Baier, S.M.; Naimo, M.A.; et al. The effects of 12 weeks of beta-hydroxy-beta-methylbutyrate free acid supplementation on muscle mass, strength, and power in resistance-trained individuals: A randomized, double-blind, placebo-controlled study. Eur. J. Appl. Physiol. 2014, 114, 1217-1227. [CrossRef] [PubMed]

37. Thomson, J.S.; Watson, P.E.; Rowlands, D.S. Effects of nine weeks of beta-hydroxy-beta- methylbutyrate supplementation on strength and body composition in resistance trained men. J. Strength Cond. Res. 2009, 23, 827-835. [CrossRef]

38. Tritto, A.C.; Bueno, S.; Rodrigues, R.M.P.; Gualano, B.; Roschel, H.; Artioli, G.G. Negligible Effects of beta-Hydroxy-beta-Methylbutyrate Free Acid and Calcium Salt on Strength and Hypertrophic Responses to Resistance Training: A Randomized, Placebo-Controlled Study. Int. J. Sport Nutr. Exerc. Metab. 2019, 29, 505-511. [CrossRef]

39. Stahn, A.C.; Maggioni, M.A.; Gunga, H.C.; Terblanche, E. Combined protein and calcium beta-hydroxy-beta-methylbutyrate induced gains in leg fat free mass: A double-blinded, placebo-controlled study. J. Int. Soc. Sports Nutr. 2020, 17, 16. [CrossRef]

40. Damas, F.; Phillips, S.M.; Libardi, C.A.; Vechin, F.C.; Lixandrao, M.E.; Jannig, P.R.; Costa, L.A.; Bacurau, A.V.; Snijders, T.; Parise, G.; et al. Resistance training-induced changes in integrated myofibrillar protein synthesis are related to hypertrophy only after attenuation of muscle damage. J. Physiol. 2016, 594, 5209-5222. [CrossRef]

41. Lowery, R.P.; Joy, J.M.; Rathmacher, J.A.; Baier, S.M.; Fuller, J.C., Jr.; Shelley, M.C., 2nd; Jager, R.; Purpura, M.; Wilson, S.M.; Wilson, J.M. Interaction of Beta-Hydroxy-Beta-Methylbutyrate Free Acid and Adenosine Triphosphate on Muscle Mass, Strength, and Power in Resistance Trained Individuals. J. Strength Cond. Res. 2016, 30, 1843-1854. [CrossRef]

42. Bhasin, S.; Storer, T.W.; Berman, N.; Callegari, C.; Clevenger, B.; Phillips, J.; Bunnell, T.J.; Tricker, R.; Shirazi, A.; Casaburi, R. The effects of supraphysiologic doses of testosterone on muscle size and strength in normal men. N. Engl. J. Med. 1996, 335, 1-7. [CrossRef]

43. Shirato, M.; Tsuchiya, Y.; Sato, T.; Hamano, S.; Gushiken, T.; Kimura, N.; Ochi, E. Effects of combined beta-hydroxy-beta-methylbutyrate (HMB) and whey protein ingestion on symptoms of eccentric exercise-induced muscle damage. J. Int. Soc. Sports Nutr. 2016, 13, 7. [CrossRef]

44. Morton, R.W.; Murphy, K.T.; McKellar, S.R.; Schoenfeld, B.J.; Henselmans, M.; Helms, E.; Aragon, A.A.; Devries, M.C.; Banfield, L.; Krieger, J.W.; et al. A systematic review, meta-analysis and meta-regression of the effect of protein supplementation on resistance training-induced gains in muscle mass and strength in healthy adults. Br. J. Sports Med. 2018, 52, 376-384. [CrossRef] [PubMed]

45. Bear, D.E.; Langan, A.; Dimidi, E.; Wandrag, L.; Harridge, S.D.R.; Hart, N.; Connolly, B.; Whelan, K. $\beta$-Hydroxy- $\beta$-methylbutyrate and its impact on skeletal muscle mass and physical function in clinical practice: A systematic review and meta-analysis. Am. J. Clin. Nutr. 2019, 109, 1119-1132. [CrossRef] [PubMed]

46. Courel-Ibáñez, J.; Vetrovsky, T.; Dadova, K.; Pallarés, J.G.; Steffl, M. Health Benefits of $\beta$-Hydroxy- $\beta$-Methylbutyrate (HMB) Supplementation in Addition to Physical Exercise in Older Adults: A Systematic Review with Meta-Analysis. Nutrients 2019, 11, 2082. [CrossRef] [PubMed] 
47. Robinson, E.H.; Stout, J.R.; Miramonti, A.A.; Fukuda, D.H.; Wang, R.; Townsend, J.R.; Mangine, G.T.; Fragala, M.S.; Hoffman, J.R. High-intensity interval training and $\beta$-hydroxy- $\beta$-methylbutyric free acid improves aerobic power and metabolic thresholds. J. Int. Soc. Sports Nutr. 2014, 11, 16. [CrossRef]

48. Durkalec-Michalski, K.; Jeszka, J.; Podgórski, T. The Effect of a 12-Week Beta-hydroxy-beta-methylbutyrate (HMB) Supplementation on Highly-Trained Combat Sports Athletes: A Randomised, Double-Blind, Placebo-Controlled Crossover Study. Nutrients 2017, 9, 753. [CrossRef]

(C) 2020 by the authors. Licensee MDPI, Basel, Switzerland. This article is an open access article distributed under the terms and conditions of the Creative Commons Attribution (CC BY) license (http://creativecommons.org/licenses/by/4.0/). 